\title{
Exosomes derived from HCC cells induce sorafenib resistance in hepatocellular carcinoma both in vivo and in vitro
}

\author{
Zhen $\mathrm{Qu}^{1,2+}$, Junhua $\mathrm{Wu}^{3 \dagger}$, Junyi $\mathrm{Wu}^{1,2}$, Dongjun Luo ${ }^{2,4}$, Chunping Jiang ${ }^{1,2^{*}}$ and Yitao Ding ${ }^{1,2^{*}}$
}

\begin{abstract}
Background: Exosomes are carriers of intercellular information and regulate the tumor microenvironment. They play an important role in drug resistance by transporting RNA molecules and proteins. However, their effects on sorafenib resistance in hepatocellular carcinoma (HCC) are not completely understood.

Methods: Exosomes were isolated from two invasive hepatoma cell lines (MHCC-97 $L$ and MHCC-97H), and their roles in regulating sorafenib resistance in liver cancer cells as well as the underlying molecular mechanisms were determined. The exosomes were analyzed by TEM (transmission electron microscopy), DLS (dynamic light scattering) and Western blotting. Cell viability, cell death and the effects of exosomes on the HGF/c-Met/Akt signaling pathway in cancer cells were analyzed by MTT assays, FACS analysis and Western blotting, respectively. Moreover, the effects of exosomes on sorafenib resistance in vivo were investigated using a subcutaneous transplantation tumor model in athymic nude mice.
\end{abstract}

Results: Exosomes derived from HCC cells were of the expected size and expressed the exosomal markers CD9 and CD63. They induced sorafenib resistance in vitro by activating the HGF/c-Met/Akt signaling pathway and inhibiting sorafenib-induced apoptosis. They also induced sorafenib resistance in vivo by inhibiting sorafenib-induced apoptosis. Moreover, exosomes derived from highly invasive tumor cells had greater efficacy than that of exosomes derived from less invasive cells.

Conclusions: These data reveal the important role of HCC cell-derived exosomes in the drug resistance of liver cancer cells and demonstrate the intrinsic interaction between exosomes and their targeted tumor cells. This study suggests a new strategy for improving the effectiveness of sorafenib in treating HCC.

Keywords: Exosomes, Sorafenib resistance, Hepatocellular carcinoma

\section{Background}

Hepatocellular carcinoma $(\mathrm{HCC})$ is the fifth most common cancer and second leading cause of cancer-related deaths worldwide, resulting in 700,000 deaths annually [1]. Currently, surgical resection is the major treatment modality for early-stage liver cancer [2]; however, most patients are diagnosed in the advanced stage when treatments have little effect. Currently, the 5-year survival rate of patients with HCC is less than $20 \%$ [3].

\footnotetext{
* Correspondence: chunpingjiang@163.com; drdingyitao0@sina.com ${ }^{\dagger}$ Equal contributors

${ }^{1}$ Department of Hepatobiliary Surgery, Affiliated Drum Tower Hospital of Nanjing University Medical School, 210008 Nanjing, Jiangsu Province, China Full list of author information is available at the end of the article
}

Phase III clinical trials showed that sorafenib, a multitarget tyrosine kinase inhibitor that decreases tumor cell proliferation and angiogenesis $[4,5]$, improved the overall survival of patients with advanced HCC [6]. Therefore, in 2007, it was approved by the US Food and Drug Administration as a molecular targeted drug for unresectable liver cancer. Although sorafenib is currently the only medication approved for the treatment of liver cancer, its therapeutic effects are affected by several signaling pathways, such as the reactivation of ERK signaling and inhibition of MAPK [7, 8]. Previous studies have suggested that gastrointestinal stromal tumor cellderived exosomes contain oncogenic KIT, and their transfer and uptake by the surrounding smooth muscle 
cells led to enhanced AKT and MAPK signaling and enhanced tumor cell invasion [9].

Exosomes play important roles in the exchange of biological information as substance transport carriers and in regulation of the cellular microenvironment by delivering a variety of biological molecules, including mRNAs, miRNAs, and proteins [10-13]. Tumor cell-derived exosomes are involved in the regulation of the epithelialmesenchymal transition, tumor angiogenesis, tumor metastasis, and radioresistance [14-17]. Docetaxel and cisplatin promote the secretion of exosomes from tumor cells; the exosomes alter drug sensitivity by releasing molecules such as mRNAs and miRNAs into neighboring cells $[18,19]$. Safei et al. [20] found that cisplatin-resistant ovarian cancer cells discharged anticancer drugs through exosomes and expressed higher levels of the transporter proteins MRP2, ATP7A and ATP7B than those of cisplatin-sensitive cells, suggesting that exosomes play a key role in resistance to chemotherapy. However, whether HCC cell-derived exosomes are involved in sorafenib resistance in liver cancer cells and the potential underlying mechanisms are currently unclear.

In this study, we investigated whether HCC cellderived exosomes mediate sorafenib resistance in HCC cells and determined the potential molecular mechanisms underlying this process. We found that HCC cellderived exosomes enhanced sorafenib resistance in liver cancer in vitro by inhibiting sorafenib-induced apoptosis via activation of the HGF/c-Met/Akt signaling pathways. These findings reveal the important role of HCC cellderived exosomes in the drug resistance of liver cancer cells and demonstrate the intrinsic interaction between exosomes and their targeted tumor cells. The results of this study may provide a new strategy for improving the effectiveness of sorafenib in treating liver cancer.

\section{Methods}

\section{Cell lines and cell culture}

The human HCC cell line SMMC-7721 was purchased from the Cell Bank of the Chinese Academy of Sciences (Shanghai, China), and the MHCC-97H, MHCC-97 L and LO2 cell lines were obtained from the Liver Cancer Institute, Fudan University (Shanghai, China). SMMC-7721, MHCC-97H and MHCC-97 L cells were cultured in complete DMEM containing $10 \%$ fetal bovine serum (FBS) and penicillin $(100 \mathrm{U} / \mathrm{mL})$. LO2 cells were cultured in RPMI-1640 medium containing $10 \%$ FBS, penicillin $(100 \mathrm{U} / \mathrm{mL})$, and streptomycin $(100 \mu \mathrm{g} / \mathrm{mL})$. All cells were incubated at $37{ }^{\circ} \mathrm{C}$ in humidified air with $5 \% \mathrm{CO}_{2}$.

\section{Extraction of exosomes}

MHCC-97H, MHCC-97 L and LO2 cell lines were cultured in media with $10 \%$ exosome-free FBS (by ultracentrifugation overnight). After $48 \mathrm{~h}$, cell culture media were collected, and exosomes were isolated from the supernatant by differential centrifugation as previously described [21]. Finally, the protein content of the concentrated exosomes was determined using a BCA protein assay kit (Thermo Scientific, USA). CD9, CD63 and GAPDH (antibodies for CD9, CD63 and GAPDH were obtained from Cell Signaling Technology, Beverly, MA, USA) expressions were measured using Western blot analyses. The aliquots were stored at $-80{ }^{\circ} \mathrm{C}$.

\section{Transmission electron microscopy (TEM) and size distribution analysis}

The extracted pellets were observed by TEM as previously described [22]. Approximately $10 \mu \mathrm{L}$ of purified exosomes was fixed in $1 \%$ glutaraldehyde for $10 \mathrm{~min}$, washed, and contrasted in $2 \%$ uranyl acetate. Images were obtained by TEM (JEM-2100, Jeol, Japan). Scanning ion occlusion sensing analysis was performed using a Zetasizer Nano ZS90 instrument (Malvern, UK) according to the manufacturer's instructions. Isolated exosome samples were resuspended in PBS. All samples were measured with parameters of $44.5 \mathrm{~mm}$ and $0.64 \mathrm{~V}$ voltage using NP100 membranes. Samples were calibrated by CPC100 standard particles diluted 1000-fold under identical settings.

\section{Animal model}

All animal procedures were performed according to national guidelines and approved by the Animal Care Ethics Committee of Nanjing Drum Tower Hospital. Twenty-five male BALB/c nu/nu mice (4-6 weeks old) were purchased from the Laboratory Animal Center of Shanghai, Academy of Science. First, all mice received subcutaneous injections of SMMC-7721 cells in the right armpit after infiltration anesthesia with lidocaine $(0.25 \%$, Nanjing Drum Tower Hospital $)\left(1 \times 10^{7}\right.$ cells in $200 \mu \mathrm{L}$ PBS per mouse). When the tumors reached a volume of $50-100 \mathrm{~mm}^{3}$ (15 days after subcutaneous injections of tumor cells), twenty-five mice were randomly divided into five groups $(n=5$, the control group, sorafenib group, sorafenib + LO2-exosome group, sorafenib + MHCC-97 L-exosome group, and sorafenib + MHCC97H-exosome group). The sorafenib group was intraperitoneally injected with sorafenib $(100 \mathrm{mg} / \mathrm{kg}$, Selleck, USA) daily for 10 days. The sorafenib + LO2-exosome group, sorafenib + MHCC-97 L-exosome group, and sorafenib + MHCC-97H-exosome group were intraperitoneally injected with sorafenib $(100 \mathrm{mg} / \mathrm{kg}$, Selleck, USA) daily and subcutaneously injected with LO2, MHCC$97 \mathrm{~L}$ and MHCC-97H cell-derived exosomes $(100 \mu \mathrm{g}$ total protein in $100 \mu \mathrm{L}$ volume, in the vicinity of the subcutaneous tumors), respectively, every day for 10 days. The control group was intraperitoneally injected with 0.4 \% dimethyl sulfoxide (DMSO) in PBS (the vehicle for 
sorafenib) and subcutaneously injected with PBS (the vehicle for exosome) daily for 10 days. The mice were examined every 2 days, and all mice sacrificed by cervical dislocation under general anesthesia with chloral hydrate $(5 \%, 100 \mu \mathrm{L} / 10 \mathrm{~g}) 25$ days after the subcutaneous injections of tumor cells.

\section{Cell viability analysis}

Cell viability was monitored using MTT assays. Briefly, $5 \times 10^{3}$ cells were cultured on 96-well plates. After incubation with sorafenib alone or sorafenib and exosomes for 24 or $48 \mathrm{~h}, 20 \mu \mathrm{L}$ MTT solution $(0.5 \%)$ was added to the medium and incubated for $4 \mathrm{~h}$. Then, the medium was removed, and $150 \mu \mathrm{L}$ DMSO was added to each well to dissolve the insoluble formazan product. The absorbance of the colored solution was measured at $490 \mathrm{~nm}$ with a spectrophotometer. All of the experiments were performed in triplicate.

\section{Western blot analysis}

HCC cells were lysed with RIPA peptide lysis buffer (Beyotime Biotechnology, China) containing $1 \%$ protease inhibitors (Thermo Scientific, USA). Equal amounts of proteins were loaded and resolved using $10 \%$ sodium dodecyl sulfate-polyacrylamide gel electrophoresis (SDSPAGE). Antibodies for CD9, CD63, GAPDH, caspase-9, caspase-3, PARP, c-Met, p-Met, AKT, p-Akt, VEGFR2 and p-VEGFR2 were obtained from Cell Signaling Technology (Beverly, MA, USA). The Met inhibitor crizotinib (PF-02341066) and the p-Akt inhibitor MK-2206 2HCl were purchased from Selleck (Selleck Chemicals, China). After incubation with horseradish peroxidase-conjugated secondary antibodies, protein bands were visualized using enhanced chemiluminescence (Millipore, USA).

\section{Fluorescence microscopy analysis of exosome internalization}

MHCC-97H-derived exosomes were labeled with CMDIL (Sigma-Aldrich, St. Louis, MO, USA) as follows. Two microliters of CM-DIL was added to $100 \mu \mathrm{g}$ of MHCC97H-derived exosomes in a total of $1 \mathrm{~mL}$ of diluent and incubated for $15 \mathrm{~min}$ at room temperature, and the mixture was added to $18 \mathrm{~mL}$ of $\mathrm{PBS}$ and centrifuged at $120,000 \mathrm{~g}$ for $2 \mathrm{~h}$ at $4{ }^{\circ} \mathrm{C}$. The supernatant was removed, and the pellet was resuspended in $20 \mathrm{~mL}$ of PBS and centrifuged at $120,000 \mathrm{~g}$ for $2 \mathrm{~h}$ at $4{ }^{\circ} \mathrm{C}$. The pellet containing CM-DIL-labeled exosomes was resuspended in $200 \mu \mathrm{L}$ of PBS medium. SMMC-7721-GFP cells were cultured in a four-chamber slide to $80 \%$ confluency. The medium was added with PBS medium containing CM-DIL-labeled exosomes, and cells were incubated for $4 \mathrm{~h}$ at $37{ }^{\circ} \mathrm{C}$ in $5 \%$ $\mathrm{CO}_{2}$. After incubation, the cells were washed twice with PBS and fixed in polyformaldehyde for $10 \mathrm{~min}$. The slide was mounted with ProLong Gold Antifade Reagents, and internalization of the exosomes was analyzed using fluorescence microscopy.

\section{TUNEL assay}

Subcutaneous tumor samples from nude mice were paraffin-sectioned by routine methods. Apoptotic cells were visualized using terminal deoxynucleotidyl transferase-mediated dUTP nick end labeling (TUNEL) assays. The TUNEL procedure was performed using an in situ cell death detection kit (Keygentec, KGA7025, China) according to the manufacturer's instructions. Briefly, the fixed cells were incubated in $20 \mathrm{mg} / \mathrm{mL}$ proteinase $\mathrm{K}$ for $30 \mathrm{~min}$ at room temperature, followed by incubation with equilibration buffer for $10 \mathrm{~s}$. The cells were washed with PBS and incubated with streptavidinFITC at $37{ }^{\circ} \mathrm{C}$ for $1 \mathrm{~h}$ in a humidified chamber. Then, the cells were washed with PBS again and incubated with anti-digoxigenin conjugate (rhodamine antibody) and counterstained with DAPI. The microscopic images of the cells were visualized by fluorescence microscopy (BX41, Olympus, Japan).

\section{Enzyme-linked immunosorbent assay (ELISA)}

The concentration of hepatocyte growth factor (HGF) in the cellular supernatant was detected using a human HGF ELISA kit (ExCell, Shanghai, China) following the manufacturer's instructions. Briefly, after the cells were treated with sorafenib and HCC cell-derived exosomes for $48 \mathrm{~h}$, the media was collected and centrifuged at $5000 \mathrm{rpm}$ for $5 \mathrm{~min}$. Total media with $10 \%$ FBS was used as the control.

\section{Statistical analysis}

All data are expressed as the mean \pm SD from three individual experiments. Differences between groups were determined using Student's $t$-test or analysis of variance (ANOVA). $P$ values less than 0.05 were considered statistically significant.

\section{Results \\ Extraction and characterization of HCC cell-derived exosomes}

To determine the effects of exosomes from different sources on sorafenib resistance in HCC cells, we first used ultracentrifugation to isolate exosomes from the supernatants of two hepatoma cell lines (MHCC-97H and MHCC-97 L) with different invasive potential and a non-invasive immortalized liver cell line (LO2). MHCC$97 \mathrm{H}$ has a higher invasive potential than that of MHCC$97 \mathrm{H}$, and LO2 is a normal non-invasive liver cell line [23]. The exosomes were round in shape with diameters of $40-150 \mathrm{~nm}$, as determined by TEM and DLS (NanoZS90, Malvern) (Fig. 1a, b), and expressed the exosomal markers CD9 and CD63 (Fig. 1c). 


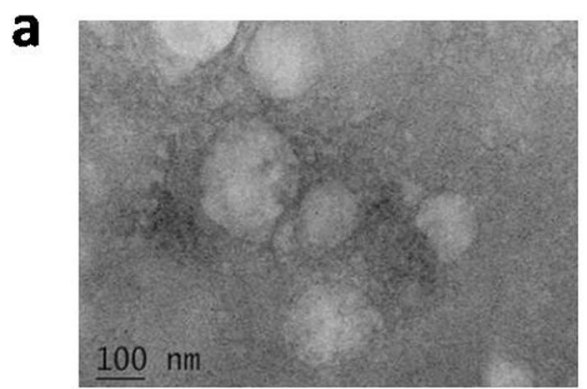

LO2-exosome

b

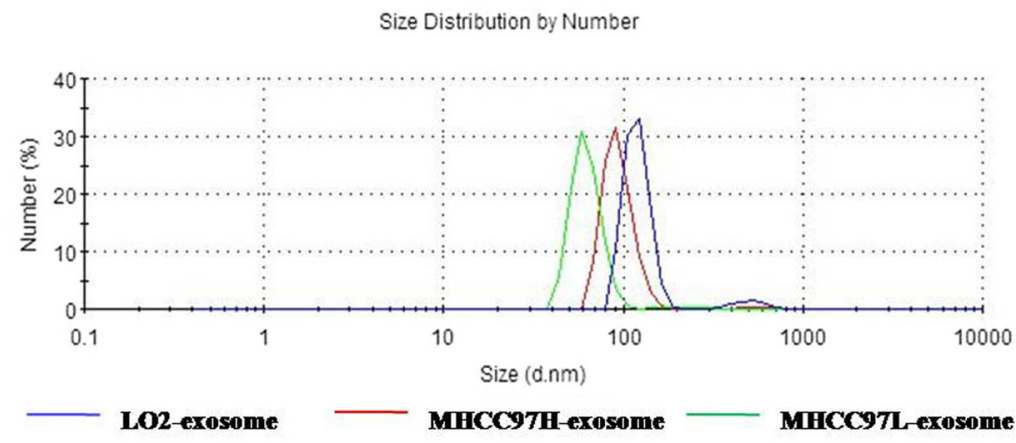

\begin{tabular}{cc}
\hline Parental cell & Exosome diameter $(\mathrm{nm})$ \\
\hline LO2 & $124.4 \pm 3.2$ \\
MHCC-97L & $60.1 \pm 1.7$ \\
MHCC-97H & $97.5 \pm 2.5$
\end{tabular}

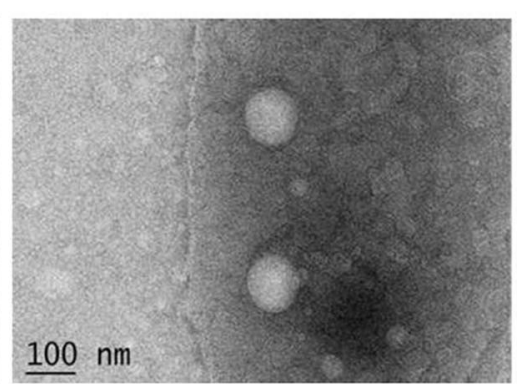

MHCC97L-exosome

C

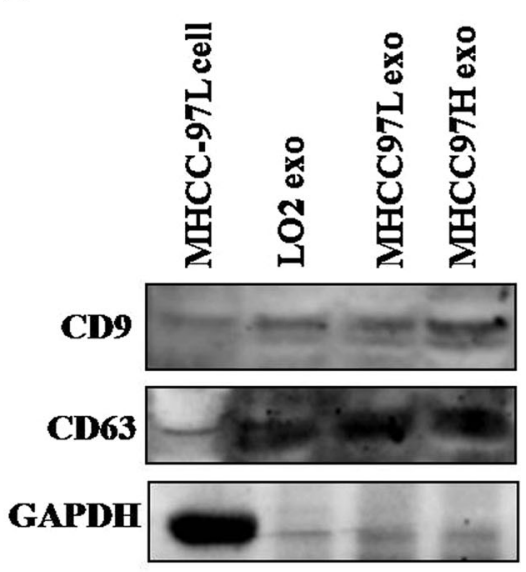

Fig. 1 Characterization of exosomes derived from different cell lines. a TEM confirmed that the final pellets from ultracentrifugation were exosomes (scale bar, $100 \mathrm{~nm}$ ). b Size distribution analysis of purified exosomes by DLS (Nano-ZS90, Malvern). c Exosomal markers (CD9, CD63) were analyzed using Western blotting and are present in cells and exosomes (GAPDH was used as an internal reference)

HCC cell-derived exosomes can be taken up and internalized by hepatoma cells

To examine the potential uptake and internalization of exosomes by SMMC-7721 cells, we labeled exosomes derived from $\mathrm{MHCC}-97 \mathrm{H}$ cells with a fluorescent dye, CMDIL, as described in Materials and Methods. CM-DILlabeled exosomes were incubated with SMMC-7721-GFP cells for $4 \mathrm{~h}$, and localization of the exosomes was assessed by fluorescence microscopy (Fig. 2). CM-DIL-labeled exosomes were internalized as endosome-like vesicles in the cytoplasm of SMMC-7721-GFP cells (Fig. 2c, d). These studies indicate that $\mathrm{HCC}$ cell-derived exosomes can be taken up and internalized by HCC cells.

HCC cell-derived exosomes induce sorafenib resistance in hepatoma cells in vivo

To determine whether HCC cell-derived exosomes can induce sorafenib resistance in liver cancer in vivo, we established a subcutaneous xenograft model in nude mice and injected sorafenib together with LO2-, MHCC-97 L-, or
MHCC-97H-derived exosomes into the mice. As shown in Fig. 3a, the tumors in mice treated with sorafenib plus MHCC-97 L- or MHCC-97H-derived exosomes were significantly larger than those in mice treated with sorafenib alone or sorafenib plus LO2-derived exosomes, indicating that invasive $\mathrm{HCC}$ cell-derived exosomes inhibit the therapeutic effects of sorafenib and promote tumor growth. Figure 3b-c shows the tumor volume and weight of each group. The tumor volume and weight of mice treated with sorafenib plus exosomes derived from MHCC-97H cells were approximately 5-fold greater than those in mice treated with sorafenib alone (Fig. 3b, c). Fig. $3 \mathrm{c}$ also demonstrates that tumors in mice treated with sorafenib plus MHCC-97H-derived exosomes were significantly larger than those in mice treated with sorafenib plus MHCC-97 L-derived exosomes, indicating that exosomes derived from a more invasive HCC cell line showed greater inhibition of the chemotherapeutic effects of sorafenib and stronger promotion of tumor growth that those of exosomes derive from less invasive cell lines. 

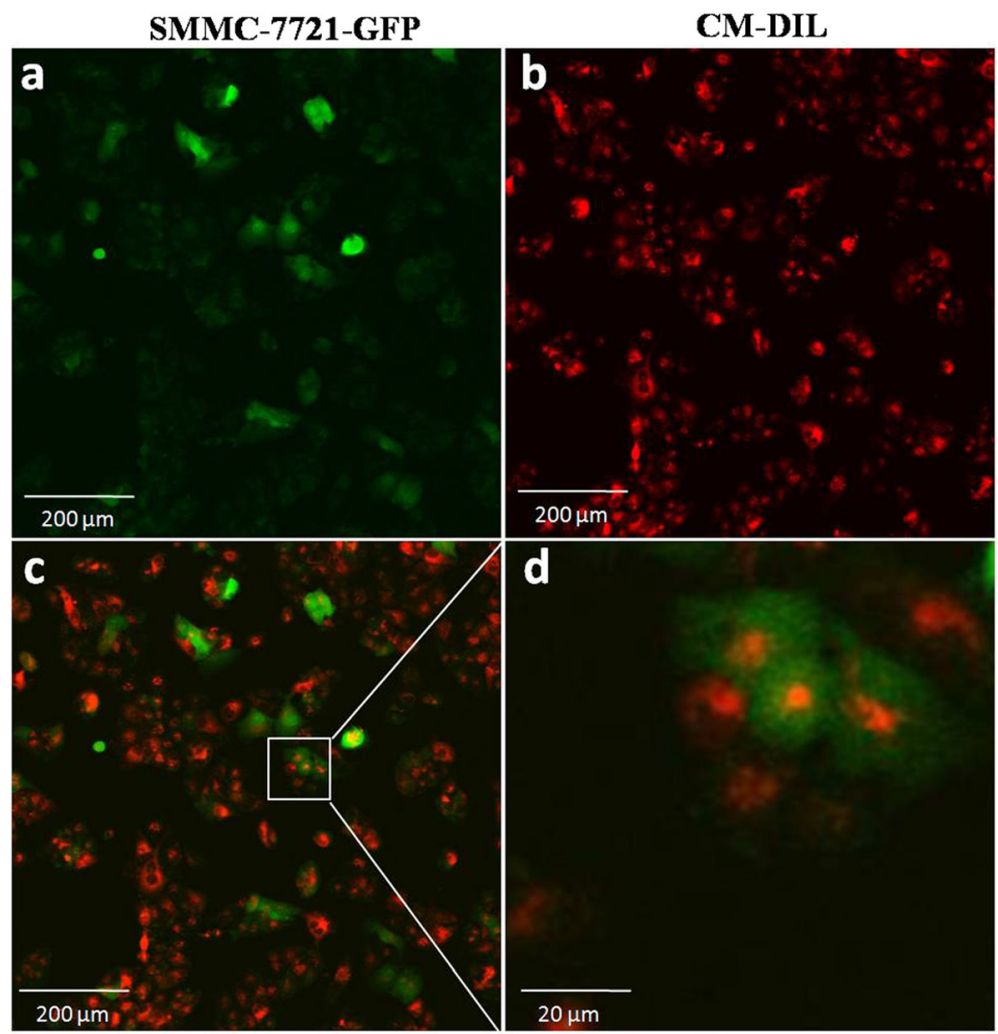

\section{Merge}

Merge

Fig. 2 Internalization of MHCC-97H-derived exosomes in SMMC-7721-GFP cells. SMMC-7721-GFP cells in culture were incubated with

MHCC-97H-derived exosomes labeled with CM-DIL (red). Cells were fixed with polyformaldehyde and mounted with ProLong Gold Antifade Reagent, as described in Materials and Methods. Low-magnification images of SMMC-7721-GFP cells incubated with exosomes (a, b, $\mathbf{c}$ ). High-magnification images of SMMC-7721-GFP cells incubated with exosomes (d). MHCC-97H-derived exosomes were shown to be internalized in the cytoplasm of SMMC-7721-GFP cells
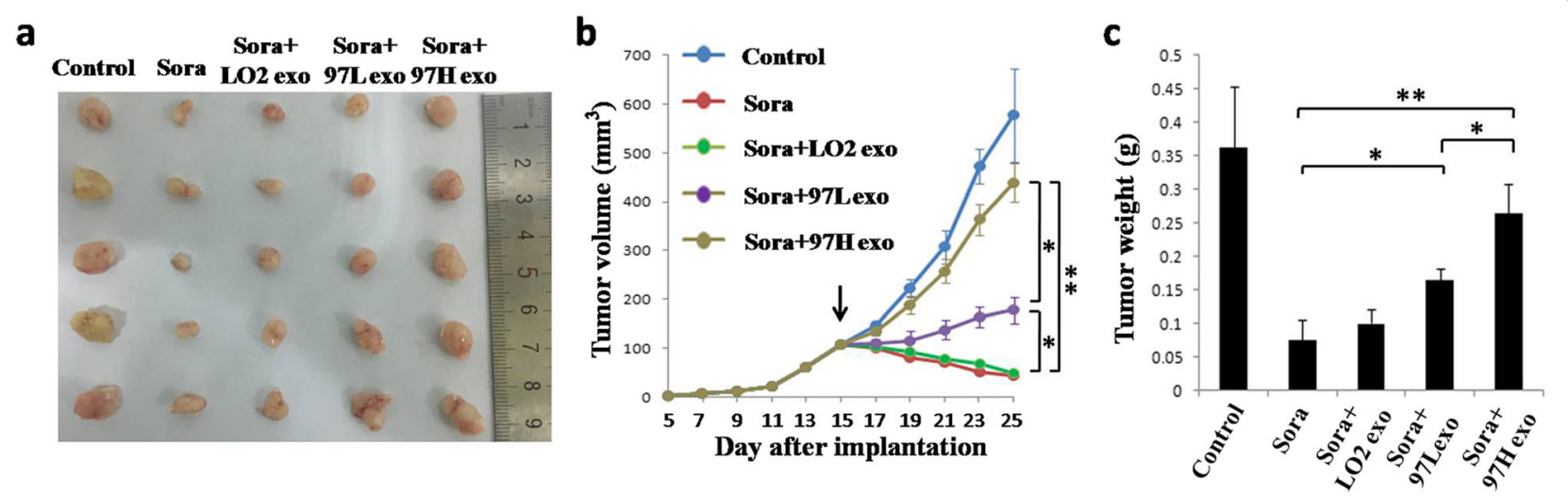

Fig. 3 HCC cell-derived exosomes induce resistance to sorafenib in hepatoma carcinoma cells in vivo. a Tumors from mice treated with PBS (Control), sorafenib (Sora), sorafenib + LO2-exosomes (Sora + LO2 exo), sorafenib + MHCC-97 L-exosomes (Sora + 97 L exo), and sorafenib + MHCC-97H-exosomes (Sora $+97 \mathrm{H}$ exo) at the end of the experiment. b Tumor growth curves in mice treated with PBS (Control), sorafenib (Sora), sorafenib + LO2-exosomes (Sora + LO2 exo), sorafenib + MHCC-97 L-exosomes (Sora + 97 L exo), and sorafenib + MHCC-97H-exosomes (Sora + 97H exo) $(n=5, * P<0.05, * * P<0.01)$. Treatment was initiated when tumors reached a volume of $50-100 \mathrm{~mm}^{3}$ (15 days after subcutaneous injections of tumor cells). $\mathbf{c}$ The mean weight of the tumors from mice treated with PBS (Control), sorafenib (Sora), sorafenib + LO2-exosomes (Sora + LO2 exo), sorafenib + MHCC-97 L-exosomes (Sora $+97 \mathrm{~L}$ exo), and sorafenib + MHCC-97H-exosomes (Sora $+97 \mathrm{H}$ exo) at the end of the experiment $(n=5, * P<0.05, * * P<0.01)$ 
Tumor volume or tumor weight in mice treated with sorafenib alone showed no significant difference from that in mice treated with sorafenib plus LO2-derived exosomes.

HCC cell-derived exosomes induce sorafenib resistance of hepatoma cells in vitro

We used MTT assays to determine the half maximal inhibitory concentration $\left(\mathrm{IC}_{50}\right)$ of sorafenib in different hepatoma cell lines. The results showed SMMC-7721 cells were more sensitive to sorafenib than MHCC-97 L and MHCC-97H cells, and MHCC-97H cells were the least sensitive to sorafenib among the three cell lines (Fig. 4a).

To determine if HCC cell-derived exosomes could induce sorafenib resistance in the SMMC-7721 HCC cell line in vitro, we used MTT assays to first confirm that sorafenib dose-dependently inhibited cell viability (Fig. 4b).
Then, we cultured the cells in medium supplemented with sorafenib plus exosomes from different sources and examined cell viability again. We found that the addition of MHCC-97H- or MHCC-97 L-derived exosomes significantly inhibited the sorafenib-induced reduction of cell viability, but addition of LO2-derived exosomes did not have this effect, indicating that invasive HCC cell-derived exosomes inhibit the therapeutic effects of sorafenib and promote tumor cell growth. Furthermore, this effect was more dramatic with MHCC-97H- compared to that of MHCC-97 L-derived exosomes $(P<0.05$, Fig. $4 \mathrm{~b})$, indicating that more invasive $\mathrm{HCC}$ cell-derived exosomes show stronger promotion of $\mathrm{HCC}$ cell proliferation and inhibition of the chemotherapeutic effects of sorafenib. Moreover, MTT assays showed that the $\mathrm{IC}_{50}$ of sorafenib in SMMC-7721 cells treated with MHCC-97H- or

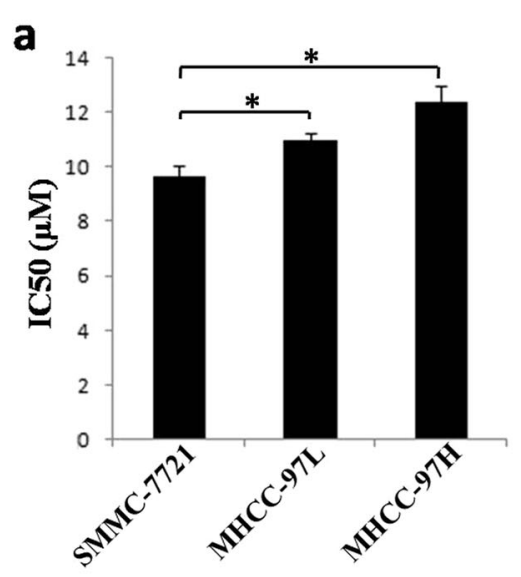

b

C

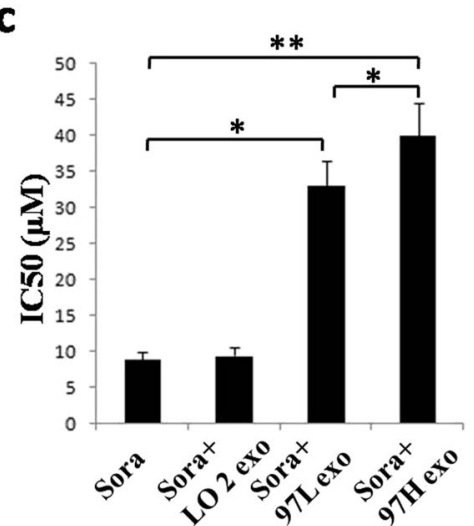

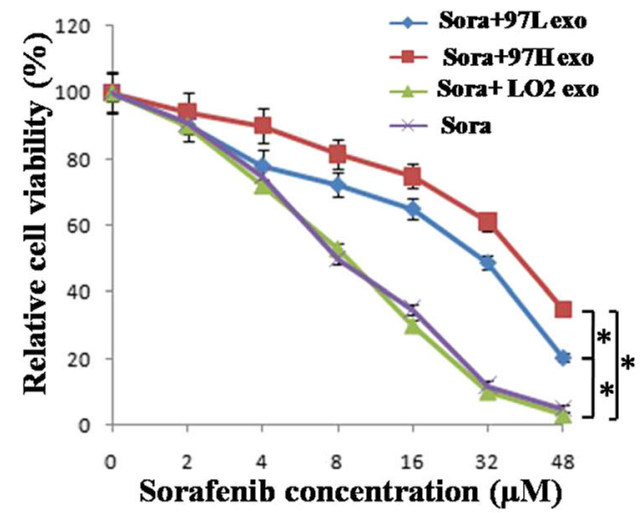

d

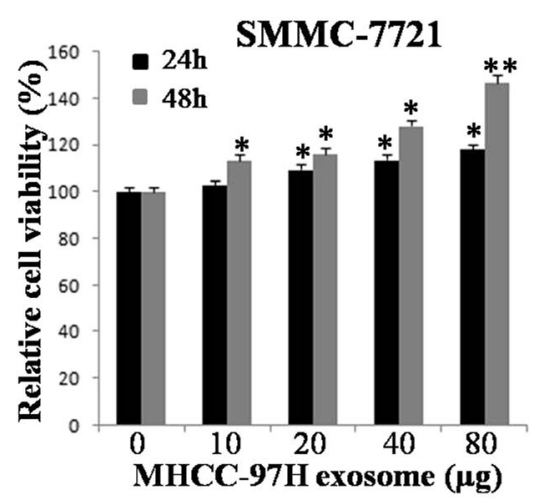

Fig. $4 \mathrm{HCC}$ cell-derived exosomes induced sorafenib resistance in SMMC-7721 cells. a IC 50 values of sorafenib in SMMC-7721, MHCC-97 L and MHCC-97H cells determined by MTT assay $(* P<0.05)$. The cells were treated with sorafenib for $48 \mathrm{~h}$, and the sensitivity of SMMC-7721 cells to sorafenib was higher than that of MHCC-97 $\mathrm{L}$ and MHCC-97H cells ( $P<0.05$ ). b Cell viability was assessed by MTT assays. HCC cell-derived exosomes attenuated sorafenib-induced cell suppression. SMMC-7721 cells were treated with sorafenib at different concentrations with or without exosomes for $48 \mathrm{~h}(* \mathrm{P}<0.05)$. $\mathbf{c} I C_{50}$ values for sorafenib in SMMC-7721 cells with or without exosomes determined by MTT assays. The SMMC-7721 cells were treated with sorafenib at different concentrations with or without exosomes for $48 \mathrm{~h}$. The IC 50 of sorafenib in SMMC-7721 cells in the HCC-derived exosome groups was notably elevated compared to that of the sorafenib alone group (* $P<0.05$; ${ }^{* *} P<0.01$ ). d MHCC-97H derived exosomes prevented the reduction of SMMC-7721 viability induced by sorafenib in a time- and dose-dependent manner (* $P<0.05$ ). Cell viability was assessed by MTT assays. The SMMC-7721 cells were treated with sorafenib at $10 \mu \mathrm{M}$ with or without exosomes at different concentrations for 24 or $48 \mathrm{~h}$ 
MHCC-97 L-derived exosomes was notably elevated compared to that in the sorafenib alone group or the LO2-exosome group, and the $\mathrm{IC}_{50}$ of sorafenib in SMMC-7721 cells treated with MHCC-97H-derived exosomes was significantly higher than that in the MHCC-97 L-derived exosome group ( $P<0.05$, Fig. 4c). Moreover, MHCC-97Hderived exosomes prevented the sorafenib-induced reduction of cell viability in a time- and dose-dependent manner (Fig. 4d).

\section{HCC cell-derived exosomes inhibit sorafenib-induced apoptosis}

To determine whether HCC cell-derived exosomes can affect sorafenib-induced cell apoptosis, we assessed sorafenib-induced apoptosis in hepatoma cells in the presence or absence of HCC cell-derived exosomes. SMMC-7721 cells were exposed to sorafenib for $48 \mathrm{~h}$, and the percentage of apoptotic cells was analyzed using Annexin V-FITC/PI apoptosis staining. The apoptotic rate of SMMC-7721 cells in the sorafenib plus MHCC97H- or MHCC-97 L-derived exosome groups was $35.42 \% \pm 2.82 \%$ and $47.33 \% \pm 7.05 \%$, respectively, which was significantly lower than that in the sorafenib alone $(60.48 \% \pm 9.42 \%)$ or sorafenib plus LO2-derived exosome groups (53.96 \% $\pm 8.64 \%$ ) (Fig. $5 \mathrm{a}, \mathrm{b}$ ).

Moreover, to further elucidate the effect of HCC cell-derived exosomes on apoptosis in vivo, we used TUNEL staining. The results demonstrated that the number of apoptotic cells in the subcutaneous tumor tissues significantly increased after tumors were treated with sorafenib alone or sorafenib plus LO2-derived exosomes (25.41 \pm $2.71,23.61 \pm 3.03$ versus $3.65 \pm 0.59$, Fig. 6a, b). However, co-treatment with HCC cell-derived exosomes significantly reduced the apoptotic rate $(10.64 \% \pm 2.44 \%$, $18.38 \% \pm 1.28 \%$ versus $25.41 \% \pm 2.71 \%$, Fig. 6a, b). Furthermore, compared to the apoptotic rate in the sorafenib plus MHCC-97 L-derived exosome group, the apoptotic rate in the sorafenib plus MHCC-97H-derived exosome group was significantly higher (Figs. $5 \mathrm{~b}$ and $6 \mathrm{~b}$ ).

Then, we assessed the effects of sorafenib alone or sorafenib plus exosomes from different sources on the levels of apoptotic proteins, such as cleaved caspase-9, caspase-3, and PARP, in SMMC-7721 cells. Treatment with sorafenib plus MHCC-97H- or MHCC-97 L-derived exosomes significantly reduced the levels of cleaved caspase-9, caspase-3, and PARP compared with
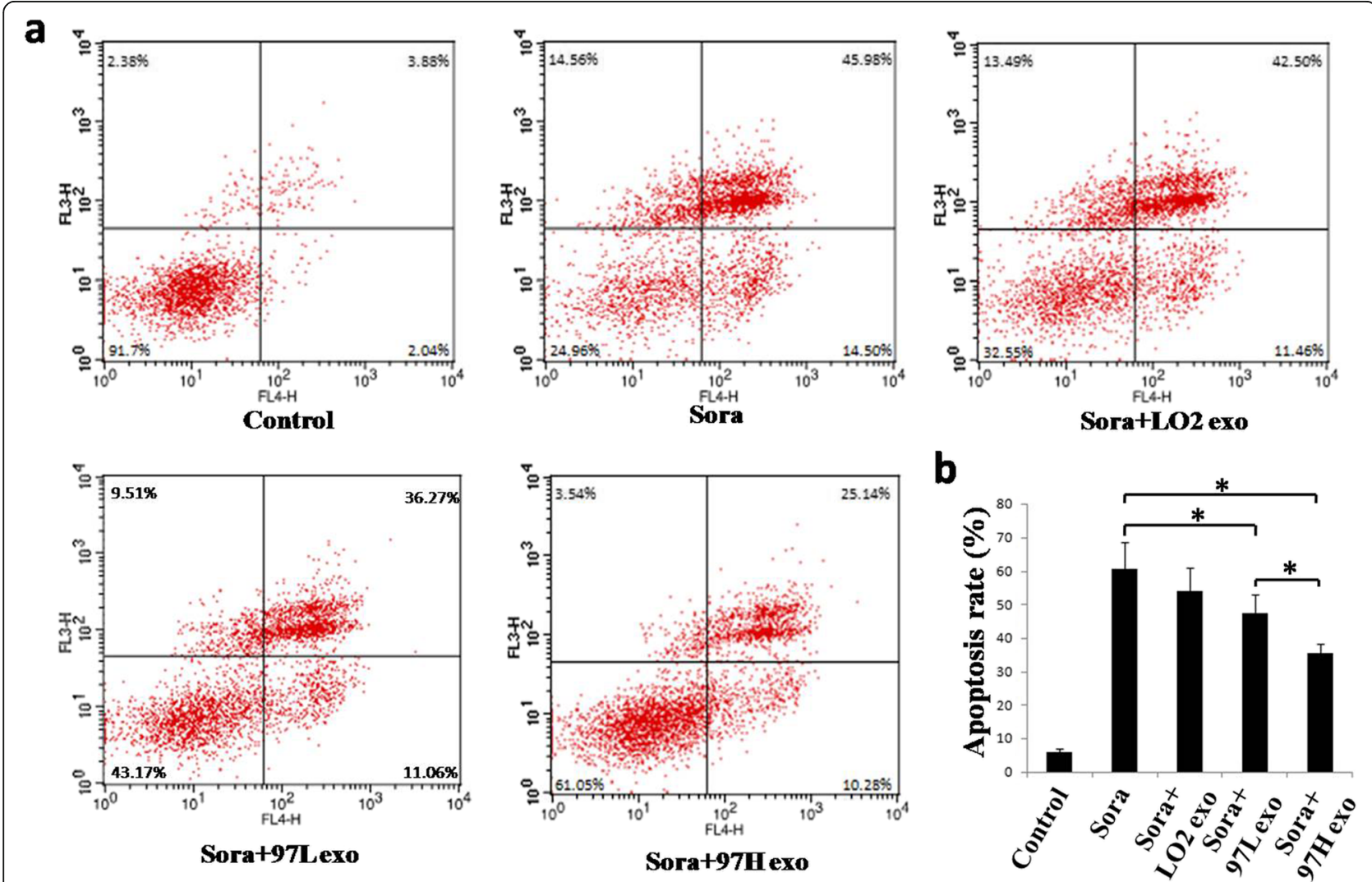

Fig. 5 HCC cell-derived exosomes reverse sorafenib-induced apoptosis in hepatoma carcinoma cells in vitro. a Flow cytometric analyses of apoptotic cells ex vivo. The SMMC-7721 cells were exposed to sorafenib alone or sorafenib and exosomes for $48 \mathrm{~h}$, collected and subjected to Annexin V/PI double staining, followed by FACS analyses. For each assay, 10,000 cells were analyzed. $\mathbf{b}$ The quantitative data are presented as the mean \pm SD of triplicate experiments $\left({ }^{*} P<0.05\right)$ 


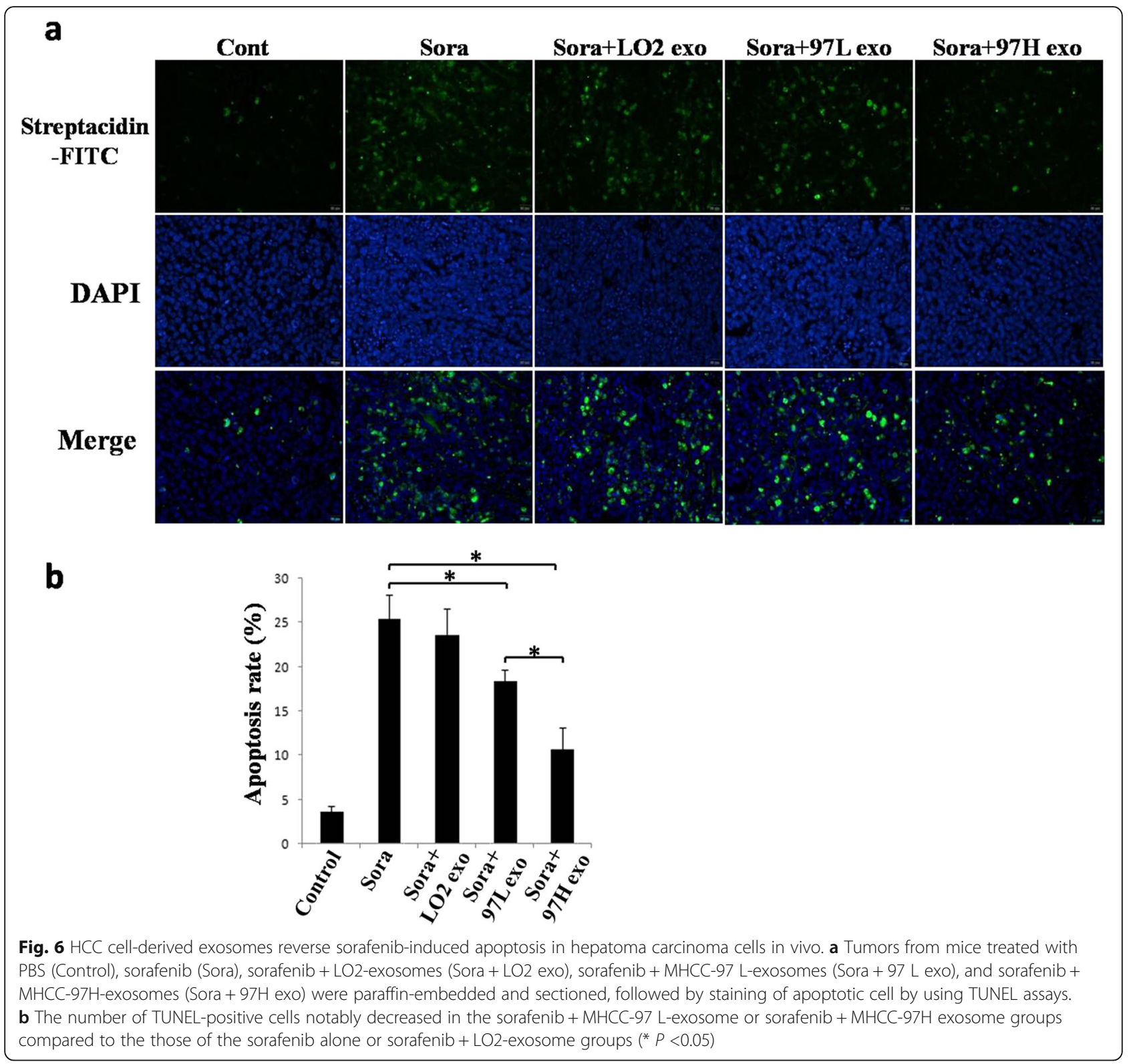

that in the sorafenib alone group or sorafenib plus LO2exosome group (Fig. 7), indicating that HCC cell-derived exosomes can partially reverse sorafenib-induced apoptosis. Interestingly, the effects of MHCC-97H-derived exosomes were more dramatic than those of MHCC97 L-derived exosomes. Taken together, these results suggested that HCC cell-derived exosomes could reverse the induction of apoptosis by sorafenib in HCC, and more invasive $\mathrm{HCC}$ cell-derived exosomes showed a greater ability to reverse sorafenib-induced apoptosis.

\section{HCC cell-derived exosomes affect sorafenib resistance via} the HGF/c-Met/Akt pathway

To explore the possible mechanisms by which HCC cell-derived exosomes induce sorafenib resistance, we also examined HGF levels in the supernatants of cells from different treatment groups using ELISAs. Previous reports have shown that exosomes can induce erlotinib resistance in breast cancer. Our results indicated that treatment with $\mathrm{HCC}$ cell-derived exosomes for $48 \mathrm{~h}$ increased HGF levels in the cell culture supernatant compared to those of the control group, and MHCC97H-derived exosomes caused the most significant effects $(P<0.05$, Fig. 8a). Western blot analysis showed that treatment with MHCC-97H- and MHCC-97 L-derived exosomes increased the levels of phosphorylated Met, Akt and VEGFR2 compared to those of the treatment with sorafenib alone or sorafenib plus LO2exosome groups (Fig. 8b). However, the increase in phosphorylated c-Met and Akt induced by MHCC-97H- 


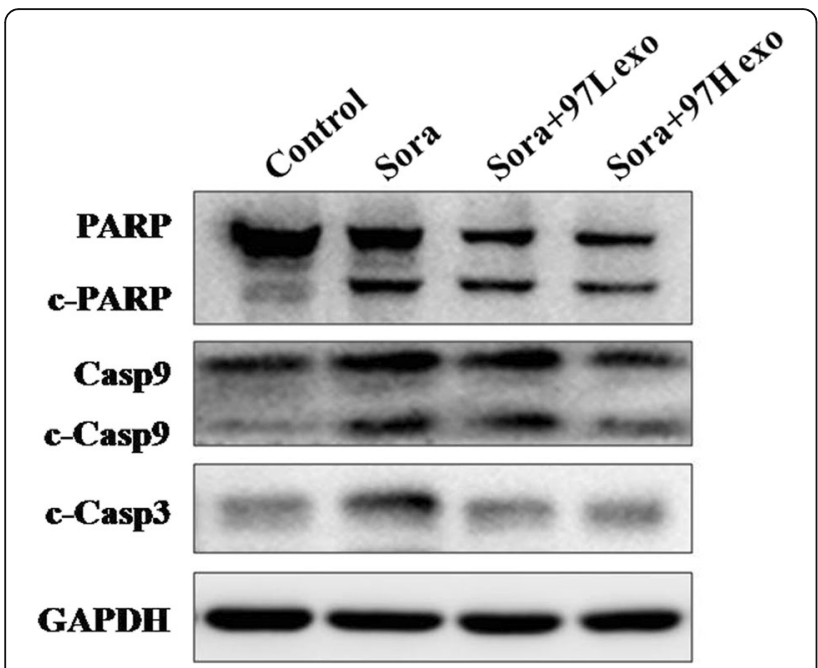

Fig. 7 HCC cell-derived exosomes decreased cleavage of caspases and PARP. MHCC-97H-derived exosomes were more effective than MHCC-97 L-exosomes. Cell lysates were subjected to Western blotting after sorafenib alone or sorafenib and exosome administration for $48 \mathrm{~h}$

and MHCC-97 L-derived exosomes was weakened by the c-Met inhibitor crizotinib (Fig. 8c), and the increase in phosphorylated Akt induced by $\mathrm{MHCC}-97 \mathrm{H}-$ and MHCC-97 L-derived exosomes was also reduced by the p-Akt inhibitor MK-2206 (Fig. 8d).

\section{Discussion}

The tumor microenvironment plays an important role in drug resistance and tumor recurrence [24, 25], which is a major obstacle to treating advanced tumors. As a transport carrier of various biological molecules, exosomes and exosomal active factors (proteins, mRNAs, miRNAs and others) participate in the regulation of the tumor microenvironment $[14,26]$. Thus, the role of exosomes as biological delivery vehicles in drug resistance is of considerable interest [27]. Previous studies suggested that a combination of guggulsterone and bexarotene reduced cellular levels of breast cancer resistance protein to $20 \%$ of that of the control cells by inducing its association and secretion with exosomes [28]. Shao et al. [29] analyzed MGMT and APNG mRNA levels in enriched tumor exosomes obtained from blood, and they found that exosomal mRNA levels of these enzymes correlated well with levels found in parental cells and that the levels changed considerably during treatment of seven glioblastoma multiforme patients. These markers may be used to predict drug response in glioblastoma multiforme patients [29]. Moreover, drug-resistant breast cancer cells may spread resistance to sensitive cells by releasing exosomes containing specific miRNAs (miR4443, miR-574-3p, and others) [30].

Exosomes derived from the serum of prostate cancer patients influenced cellular proliferation, invasion and response to docetaxel, which may be partly due to exosomal MDR-1/Pgp transfer [18]. Bone marrow stromal cells and multiple myeloma cells could mutually exchange exosomes carrying specific cytokines, which increased multiple myeloma cell growth and induced drug resistance to bortezomib [31]. In addition, exosomal lncRNA has recently attracted more attention. Takahashi et al. [32] found that specific exosomal lncRNA mediators, such as lincRNA-ROR, are involved in modulation of hepatoma cellular responses to sorafenib. Moreover, lincRNA-VLDLR could be transferred by HCC cell-derived exosomes and modulate resistance to anti-cancer agents, such as sorafenib, camptothecin, and doxorubicin, in recipient cancer cells [33]. Here, we showed for the first time that different invasive HCC cell-derived exosomes can regulate the sensitivity of HCC to sorafenib, in part by reversing sorafenib-induced apoptosis (Figs. 5, 6 and 7). Moreover, elevated expression of a cytokine (HGF) may be an important mechanisms underlying $\mathrm{HCC}$ resistance to sorafenib (Fig. 8). HCC cell-derived exosomes transfer of the soluble factor HGF may contribute to the regulation of the tumor microenvironment [34].

The HGF receptor, c-Met, is a proto-oncogene $[5,35]$. Studies have found that its overexpression in tumor cells is an important mechanism of sorafenib resistance, and the c-Met-targeting drug PHA665752 inhibits its expression in various HCC cell lines. In vivo experiments also confirmed that inhibition of c-Met in HCC cells could increase their sensitivity to therapeutic agents [36]. Our previous study found that hepatic stellate cells and their conditioned medium promoted cell proliferation and enhanced the sorafenib resistance of liver cancer cells [37]. In this study, we found that treatment of HCC cells with MHCC-97 L and MHCC-97H cell-derived exosomes increased HGF levels in the medium, which further activated p-Met in liver cancer cells, ultimately leading to activation of its key downstream protein, p-Akt (Fig. 8a, b). The effect induced by HCC cell-derived exosomes was reversed by the c-Met inhibitor crizotinib and the p-Akt inhibitor MK-2206 in recipient cells. Both inhibitors indirectly or directly reduced the phosphorylation of Akt (Fig. 8c, d). Our studies confirmed that HCC cell-derived exosomes induced sorafenib resistance by increasing HGF levels in the tumor microenvironment and activating the c-Met/Akt pathway in vitro, suggesting that $\mathrm{HGF} / \mathrm{c}-$ Met may be an important target for improving sorafenib resistance of HCC. Our study further revealed that HCC cell-derived exosomes promoted sorafenib resistance in liver cancer, and exosomes derived from highly invasive tumors could trigger stronger drug resistance. Mian et al. characterized the exosomal RNA and proteome contents derived from three HCC cell lines (HKCI-C3, HKCI-8 and MHCC- 


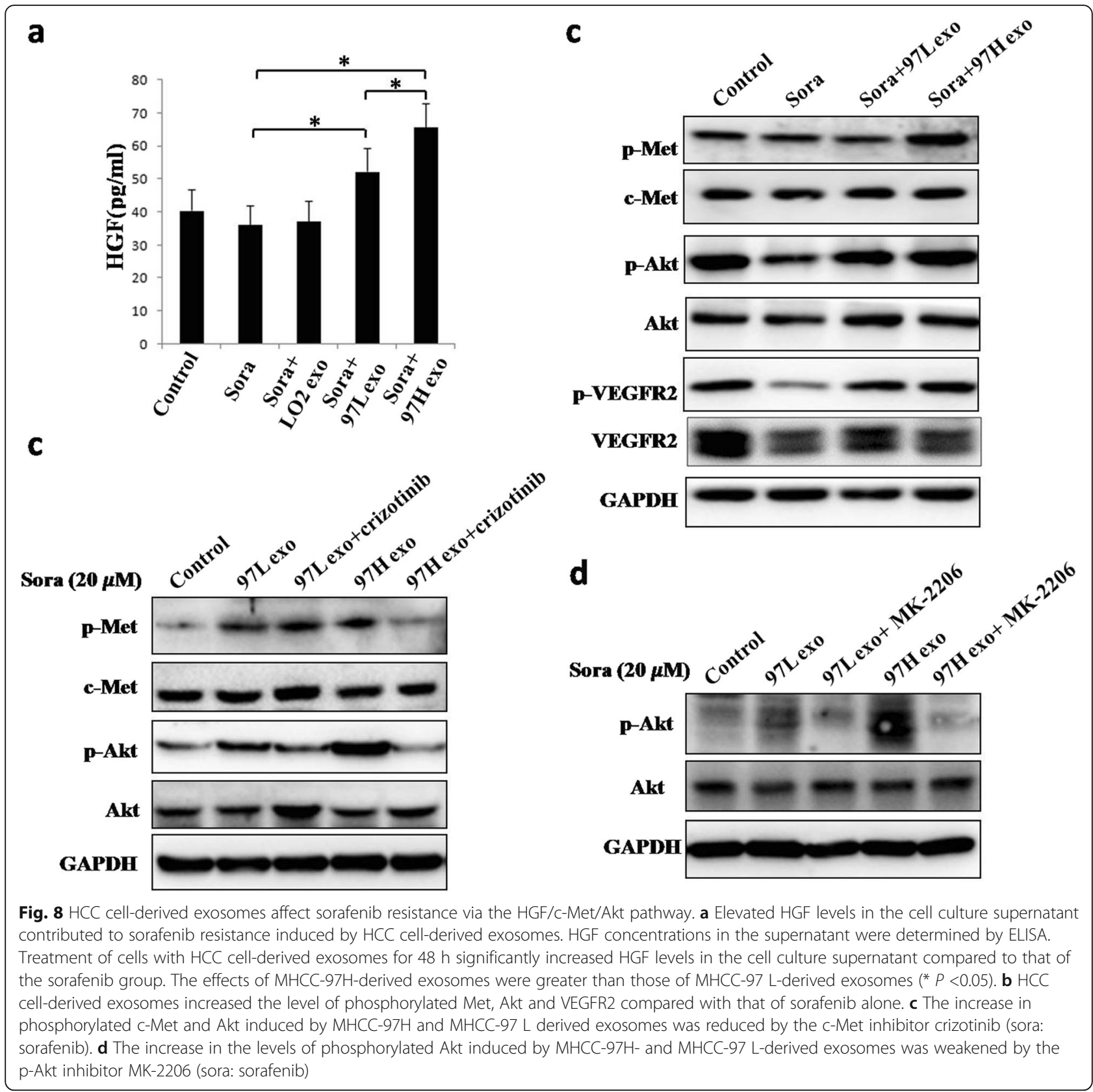

97 L) using Ion Torrent sequencing and mass spectrometry. RNA deep sequencing and proteomic analysis revealed that exosomes derived from different metastatic HCC cell lines had different levels of proteins and protumorigenic RNAs. Thus, we hypothesized that exosomes from different sources contained different levels of cytokines (HGF, TGF $\beta$, and VEGF) and proteins [34], which could be transported to recipient cells and played various roles in the regulation of sorafenib resistance in HCC. Moreover, differential miRNA expression in exosomes derived from different cell lines should be determined using miRNA microarrays, and the roles of these miRNAs in sorafenib resistance of liver cancer should be explored further.

\section{Conclusions}

In summary, this study confirmed that HCC cell-derived exosomes can enhance sorafenib resistance in liver cancer cells in vitro, and exosomes derived from highly invasive tumors have greater effects than those derived from less invasive tumors. Moreover, HCC cell-derived exosomes exerted their functions by increasing the level of proteins related to sorafenib resistance, protecting tumor cells from sorafenib-induced apoptosis and activating the HGF/c- 
Met/Akt pathway in vitro. Our results suggest that HCC cell-derived exosomes are important mediators of sorafenib resistance in liver cancer cells. Targeting HCC cell-derived exosomes or the HGF/c-Met/Akt pathway may help improve treatment efficacy in liver cancer.

\section{Acknowledgements}

We thank Translational Medicine Core facilities of Medical School of Nanjing University for instruments supporting.

\section{Funding}

The research was supported by the National Natural Science Foundation of China (no. 81300337, 81572393, 81472820, 81172143, 11334004, 81602093, 81602093 and 81421091), the National Basic Research Program of China (973 Program, 2013CB834100), the Key Project supported by Medical Science and Technology Development Foundation, Nanjing Municipality Health Bureau (no. ZKX15020 and ZKX 12011), the Natural Science Foundation of Jiangsu Province (no. BK20141324 and BK20160118), Jiangsu special program for clinical medical science and technology (BL2014054). The funding body play roles in the design of the study and execution as well as collection, analysis, and interpretation of data and in writing the manuscript.

\section{Availability of data and material}

All data generated or analysed during this study are included in this published article.

\section{Authors' contributions}

Conceived and designed the experiments: ZQ JW YD. Performed the experiments: ZQ JW. Analyzed the data: ZQ JW CJ YD. Contributed reagents/ materials/analysis tools: DL JW CJ. Wrote the paper: ZQ JW YD. Final approval of manuscript: All authors.

\section{Competing interests}

The authors declare that they have no competing interests.

\section{Consent for publication}

Not applicable.

\section{Ethics approval and consent to participate}

All animal procedures were performed according to national guidelines and approved by the Animal Care Ethics Committee of Nanjing Drum Tower Hospital.

\section{Author details}

'Department of Hepatobiliary Surgery, Affiliated Drum Tower Hospital of Nanjing University Medical School, 210008 Nanjing, Jiangsu Province, China. ${ }^{2}$ Jiangsu Province's Key Medical Center for Hepatobiliary Surgery, 210008 Nanjing, Jiangsu Province, China. ${ }^{3}$ Jiangsu Key Laboratory of Molecular Medicine, Medical School, Nanjing University, 210093 Nanjing, Jiangsu Province, China. ${ }^{4}$ Department of Hepatobiliary Surgery, Drum Tower Clinica College of Nanjing Medical University, 210008 Nanjing, Jiangsu Province, China.

Received: 21 June 2016 Accepted: 17 September 2016 Published online: 30 September 2016

\section{References}

1. Torre LA, Bray F, Siegel RL, Ferlay J, Lortet-Tieulent J, Jemal A. Global cancer statistics, 2012. CA Cancer J Clin. 2015;65:87-108.

2. El-Serag HB, Rudolph KL. Hepatocellular carcinoma: epidemiology and molecular carcinogenesis. Gastroenterol. 2007;132:2557-76.

3. El-Serag HB, Mason AC. Hepatocellular carcinoma. N Engl J Med. 2011;365:1118-27.

4. Ellis LM, Hicklin DJ. VEGF-targeted therapy: mechanisms of anti-tumour activity. Nat Rev Cancer. 2008;8:579-91.

5. Xu W, Yang Z, Lu N. Molecular targeted therapy for the treatment of gastric cancer. J Exp Clin Cancer Res. 2016;35:1.

6. Cheng AL, Kang YK, Chen Z, Tsao CJ, Qin S, Kim JS, et al. Efficacy and safety of sorafenib in patients in the Asia-Pacific region with advanced hepatocellular carcinoma: a phase III randomised, double-blind, placebocontrolled trial. Lancet Oncol. 2009;10:25-34.
7. Moritz A, Li Y, Guo A, Villén J, Wang Y, MacNeill J, et al. Akt-RSK-S6 kinase signaling networks activated by oncogenic receptor tyrosine kinases. Sci Signal. 2010;3:ra64.

8. Wilson TR, Fridlyand J, Yan Y, Penuel E, Burton L, Chan E, et al. Widespread potential for growth-factor-driven resistance to anticancer kinase inhibitors. Nature. 2012:487:505-9.

9. Atay S, Godwin AK. Tumor-derived exosomes: a message delivery system for tumor progression. Commun Integr Biol. 2014;7:e28231.

10. van der Pol E, Böing AN, Harrison P, Sturk A, Nieuwland R. Classification, functions, and clinical relevance of extracellular vesicles. Pharmacol Rev. 2012;64:676-705.

11. Regev-Rudzki N, Wilson DW, Carvalho TG, Sisquella X, Coleman BM, Rug M, et al. Cell-cell communication between malaria-infected red blood cells via exosome-like vesicles. Cell. 2013;153:1120-33.

12. Falcone G, Felsani A, D'Agnano I. Signaling by exosomal microRNAs in cancer. J Exp Clin Cancer Res. 2015;34:32.

13. Paladini L, Fabris L, Bottai G, Raschioni C, Calin GA, Santarpia L. Targeting microRNAs as key modulators of tumor immune response. J Exp Clin Cancer Res. 2016;35:103.

14. Azmi AS, Bao B, Sarkar FH. Exosomes in cancer development, metastasis, and drug resistance: a comprehensive review. Cancer Metastasis Rev. 2013;32:623-42.

15. Junttila MR, de Sauvage FJ. Influence of tumour micro-environment heterogeneity on therapeutic response. Nature. 2013;501:346-54.

16. Hamam D, Abdouh M, Gao ZH, Arena V, Arena M, Arena GO. Transfer of malignant trait to BRCA1 deficient human fibroblasts following exposure to serum of cancer patients. J Exp Clin Cancer Res. 2016;35:80.

17. Tang Y, Cui Y, Li Z, Jiao Z, Zhang Y, He Y, et al. Radiation-induced miR-208a increases the proliferation and radioresistance by targeting p21 in human lung cancer cells. J Exp Clin Cancer Res. 2016;35:7.

18. Corcoran C, Rani S, O'Brien K, O'Neill A, Prencipe M, Sheikh R, et al. Docetaxelresistance in prostate cancer: evaluating associated phenotypic changes and potential for resistance transfer via exosomes. PLoS One. 2012;7:e50999.

19. Xiao X, Yu S, Li S, Wu J, Ma R, Cao H, et al. Exosomes: decreased sensitivity of lung cancer A549 cells to cisplatin. PLoS One. 2014:9:e89534.

20. Safaei R, Larson BJ, Cheng TC, Gibson MA, Otani S, Naerdemann W, et al. Abnormal lysosomal trafficking and enhanced exosomal export of cisplatin in drug-resistant human ovarian carcinoma cells. Mol Cancer Ther. 2005:4:1595-604.

21. Valadi H, Ekström K, Bossios A, Sjöstrand M, Lee JJ, Lötvall JO. Exosomemediated transfer of mRNAs and microRNAs is a novel mechanism of genetic exchange between cells. Nat Cell Biol. 2007;9:654-9.

22. Lässer C, Eldh M, Lötvall J. Isolation and characterization of RNA-containing exosomes. J Vis Exp. 2012;9:e3037.

23. Tian J, Tang ZY, Ye SL, Liu YK, Lin ZY, Chen J, et al. New human hepatocellular carcinoma (HCC) cell line with highly metastatic potentia (MHCC97) and its expressions of the factors associated with metastasis. Br J Cancer. 1999;81:814-21.

24. Guan J, Chen J. Mesenchymal stem cells in the tumor microenvironment. Biomed Rep. 2013;1:517-21.

25. Westover D, Li F. New trends for overcoming ABCG2/BCRP-mediated resistance to cancer therapies. J Exp Clin Cancer Res. 2015;34:159.

26. Ma R, Jiang $T$, Kang $X$. Circulating microRNAs in cancer: origin, function and application. J Exp Clin Cancer Res. 2012;31:38.

27. Zhang X, Yuan X, Shi H, Wu L, Qian H, Xu W. Exosomes in cancer: small particle, big player. J Hematol Oncol. 2015;8:83.

28. Kong JN, He Q, Wang G, Dasgupta S, Dinkins MB, Zhu G, et al. Guggulsterone and bexarotene induce secretion of exosome-associated breast cancer resistance protein and reduce doxorubicin resistance in MDAMB-231 cells. Int J Cancer. 2015;137:1610-20.

29. Shao H, Chung J, Lee K, Balaj L, Min C, Carter BS, et al. Chip-based analysis of exosomal mRNA mediating drug resistance in glioblastoma. Nat Commun. 2015;6:6999

30. Zhong $S$, Chen $X$, Wang D, Zhang $X$, Shen $H$, Yang $S$, et al. MicroRNA expression profiles of drug-resistance breast cancer cells and their exosomes. Oncotarget. 2016;7:19601-9.

31. Wang J, Hendrix A, Hernot S, Lemaire M, De Bruyne E, Van Valckenborgh E, et al. Bone marrow stromal cell-derived exosomes as communicators in drug resistance in multiple myeloma cells. Blood. 2014;124:555-66.

32. Takahashi K, Yan IK, Kogure T, Haga H, Patel T. Extracellular vesicle-mediated transfer of long non-coding RNA ROR modulates chemosensitivity in human hepatocellular cancer. FEBS Open Bio. 2014;4:458-67. 
33. Takahashi K, Yan IK, Wood J, Haga H, Patel T. Involvement of extracellular vesicle long noncoding RNA (linc-VLDLR) in tumor cell responses to chemotherapy. Mol Cancer Res. 2014;12:1377-87.

34. Chowdhury R, Webber JP, Gurney M, Mason MD, Tabi Z, Clayton A. Cancer exosomes trigger mesenchymal stem cell differentiation into proangiogenic and pro-invasive myofibroblasts. Oncotarget. 2015;6:715-31.

35. Eger A, Stockinger A, Park J, Langkopf E, Mikula M, Gotzmann J, et al. Betacatenin and TGFbeta signalling cooperate to maintain a mesenchymal phenotype after FosER-induced epithelial to mesenchymal transition. Oncogene. 2004;23:2672-80.

36. You H, Ding W, Dang H, Jiang Y, Rountree CB. C-Met represents a potential therapeutic target for personalized treatment in hepatocellular carcinoma. Hepatology. 2011;54:879-89.

37. Chen W, Wu J, Shi H, Wang Z, Zhang G, Cao Y, et al. Hepatic stellate cell coculture enables sorafenib resistance in Huh7 cells through HGF/c-Met/Akt and Jak2/Stat3 pathways. BioMed Res Int. 2014;2014:764981.

Submit your next manuscript to BioMed Central and we will help you at every step:

- We accept pre-submission inquiries

- Our selector tool helps you to find the most relevant journal

- We provide round the clock customer support

- Convenient online submission

- Thorough peer review

- Inclusion in PubMed and all major indexing services

- Maximum visibility for your research

Submit your manuscript at www.biomedcentral.com/submit
Biomed Central 